\title{
Protein metabolism, physicochemical properties and chemical composition of muscle tissue in Large White weaners
}

\author{
V. Khalak', A. Horchanok², O. Kuzmenko ${ }^{3}$, L. Lytvyshchenko², M. Lieshchova², \\ A. Kalinichenko ${ }^{2}$, V. Liskovich ${ }^{3}$, L. Zagoruy ${ }^{3}$ \\ ${ }^{1}$ Institute of Grain Crops of National Academy of Agrarian Sciences of Ukraine, \\ 14 Volodymyr Vernadskii St, Dnipro, Ukraine, 49027 \\ ${ }^{2}$ Dnipro State Agrarian and Economic University, 25 Serhii Yefremov St, Dnipro, Ukraine, 49027 \\ ${ }^{3}$ Bila Tserkva National Agrarian University, 8/1Cathedral Sguare St, Bila Tserkva, Ukraine, 09117
}

*Corresponding author E-mail: v16kh91@gmail.com
Received: 07.08.2020. Accepted: 17.09.2020

\begin{abstract}
We presented the blood serum biochemical parameters, physicochemical properties and chemical composition of muscle tissue in Large White weaners of British origin. We found that serum biochemical parameters in five months weaners were within the physiological norm (the content of total protein was $71.28 \mathrm{~g} / \mathrm{l}(\mathrm{CV}=8.16 \%)$, creatinine concentration was $155.62 \mu \mathrm{mol} \mathrm{L}^{-1}(\mathrm{CV}=$ 14.13\%). Studies of physicochemical properties and chemical composition of samples of Longissinus dorsi muscle of Large White young pigs show that the number of high quality samples in terms of moisture holding capacity, $\%$ is $12.0 \%$, colour intensity, units of ext. $\times 1000-16.0 \%$, tenderness, $s-12.0 \%$ and fat content, $\%-16.0 \%$. We revealed significant correlations between moisture holding capacity and protein content $(r=-0.484, \operatorname{tr}=2.65)$, moisture holding capacity and hygroscopic moisture content $(r=0.402, t r=2.11)$. The correlation between total protein content in blood serum and fat content in muscle tissue was -0.257 $(\operatorname{tr}=1.28)$, whereas the correlation between serum total protein content and calcium content in muscle tissue was 0.375 (tr $=$ 1.94).
\end{abstract}

Key words: weaners, breed, protein metabolism, blood serum, physicochemical properties, chemical composition, muscle tissue, correlation.

\section{Introduction}

Modern pig farming is based on improving the conditions of keeping and feeding pigs of different classes. In Ukraine, the assessing of pig meat productivity, reproductive qualities of sows and boars, fattening and meat qualities of their offspring have been studied by Bazhov \& Komlatskiy (1989), Kabanov (2009), Tatulov (2009), Rybalko et al., (2010), Fedorenkova et al., (2012), Aknevskyy et al., (2013), Bankovskaja \& Voloschuk (2015), Balatskii et al., (2015, 2018), and Khalak et al., (2020a). The problems of early prediction of fattening, slaughter and meat qualities of pigs, physicochemical properties and chemical composition of muscle tissue and blubber are pf great importance and being studied by domestic and foreign scientists (Eidrigevich \& Rayevskaya, 1966; Christianson, 1991; Allison \& Laven, 2000; Levchenko, 2002; Berezovskyy \& Khatko, 2005; Tatulov, 2009; Susol, 2015; Tserenyuk, 2017, 2018; Kramarenko et al., 2018, 2019; Khalak et al., 2020b).

The aim of the work is to study physicochemical properties and chemical composition of muscle tissue in Large White weaners taking into account class of their distribution by total protein content and serum creatinine concentration, as well as to calculate the level of correlations between the main quantitative traits.

\section{Material and methods}

The study was conducted at breeding farm for Large White pigs of LLC "AF "Dzerzhynets" (Dnipropetrovsk region, Scientific Research Centre of Biosafety and Environmental Control of Resources, Agro-Industrial Complex of the Dnipro State Agrarian and Economic University, LLC "Hlobinskii miasokombinat", Poltava region, Laboratory of Zoochemical Analysis of the Institute of Big Breeding and Agro-Industrial Production of National Academy of Agrarian Sciences, Ukraine, and Laboratory of Animal Husbandry of the State Establishment Institute of Grain Crops of National Academy of Agrarian Sciences, Ukraine. The work was performed according to the research program of National Academy of Agrarian Sciences No 30 "Pig Breeding".

The formation of experimental groups was carried out according to their distribution into classes due to the content of total protein and the creatinine concentration in the blood serum of weaners. The deviation from the arithmetic mean of the trait 
was equal to $\pm 0.67 \sigma$. Thus, in weaners of class $\mathrm{M}^{+}$total protein content was $77.53 \mathrm{~g} / \mathrm{l}$ and more, creatinine concentration $171.06 \mu \mathrm{mol} / \mathrm{l}$ and more. In animals of the opposite class (M-) these values ranged from 58.67 to $66.30 \mathrm{~g} / \mathrm{l}$ and 102.59 $137.86 \mu \mathrm{mol} / \mathrm{l}$, respectively.

Blood sampling from animals of the experimental group $(n=25)$ was performed at the age of five months. Serum was tested for total protein $\left(\mathrm{g} \mathrm{L}^{-1}\right)$ and creatinine concentration ( $\mu \mathrm{mol} \mathrm{L} \mathrm{L}^{-1}$ ) (Vlyzlo et al., 2012; Hryban at al., 2001; Kamyshnikov, 2002). Control fattening of young pigs was carried out according to requirements of modern research methods in pig breeding (Methods of assessment of boars and sows for..., 2005). We determined the $\mathrm{pH}$ - active acidity, moisture holding capacity, colour intensity, tenderness, loss during thermal processing, energy value of muscle tissue, hygroscopic moisture content, airdry matter, total moisture content, crude ash, crude fat, crude protein, calcium and phosphorus content (Metodicheskiye rekomendatsii po otsenke ..., 1987, Polivoda et al., 1977, Polyvoda, 1976).

A comprehensive assessment of meat quality was determined according to Polyvoda (1976) (Table 1).

Table 1. Meat assessing scale

\begin{tabular}{|c|c|c|c|c|c|}
\hline Grade & $\begin{array}{c}\text { moisture holding } \\
\text { capacity, } \%\end{array}$ & $\begin{array}{l}\text { colour intensity, } \\
(\text { extinction } \\
\text { coefficient } \times 1000)\end{array}$ & tenderness, sec & fat, $\%$ & $\begin{array}{l}\text { melting point of } \\
\text { blubber, degrees }\end{array}$ \\
\hline Limits & $46.8-71.8$ & $27-119$ & $5.8-15.5$ & $0.7-4.8$ & $23.5-46.8$ \\
\hline High quality & $\geq 67.0$ & $\geq 83$ & $\leq 7.9$ & $\geq 3.1$ & - \\
\hline Standard quality & $53.0-66.0$ & $48-82$ & $8.0-12.0$ & $1.2-3.0$ & $32.5-41.5$ \\
\hline Low quality & $\leq 52.0$ & $\leq 47$ & $\geq 12.1$ & $\leq 1.1$ & $\begin{array}{l}\geq 41.6 \\
\leq 32.4\end{array}$ \\
\hline
\end{tabular}

\section{Results and discussion}

The analysis of the obtained data shows that total protein and creatinine in the blood serum in weaners from experimental group were within the physiological norm (Table 2).

Table 2. Biochemical parameters of blood serum of young pigs in the experimental group, $n=25$.

\begin{tabular}{lcccc}
\hline Parameter & Norm & \multicolumn{3}{c}{ Biometric indicators } \\
& & mean \pm SD & $\sigma \pm S \sigma$ & $\mathrm{CV} \pm \mathrm{Scv} . \%$ \\
\hline Total protein, $\mathrm{g} \mathrm{L}^{-1}$ & $79.0-89.0$ & $71.28 \pm 1.16$ & $5.82 \pm 0.82$ & $8.16 \pm 1.15$ \\
Creatinine, $\mu \mathrm{mol} \mathrm{L}^{-1}$ & $140-240$ & $155.62 \pm 4.39$ & $21.99 \pm 3.11$ & $14.13 \pm 1.99$ \\
\hline
\end{tabular}

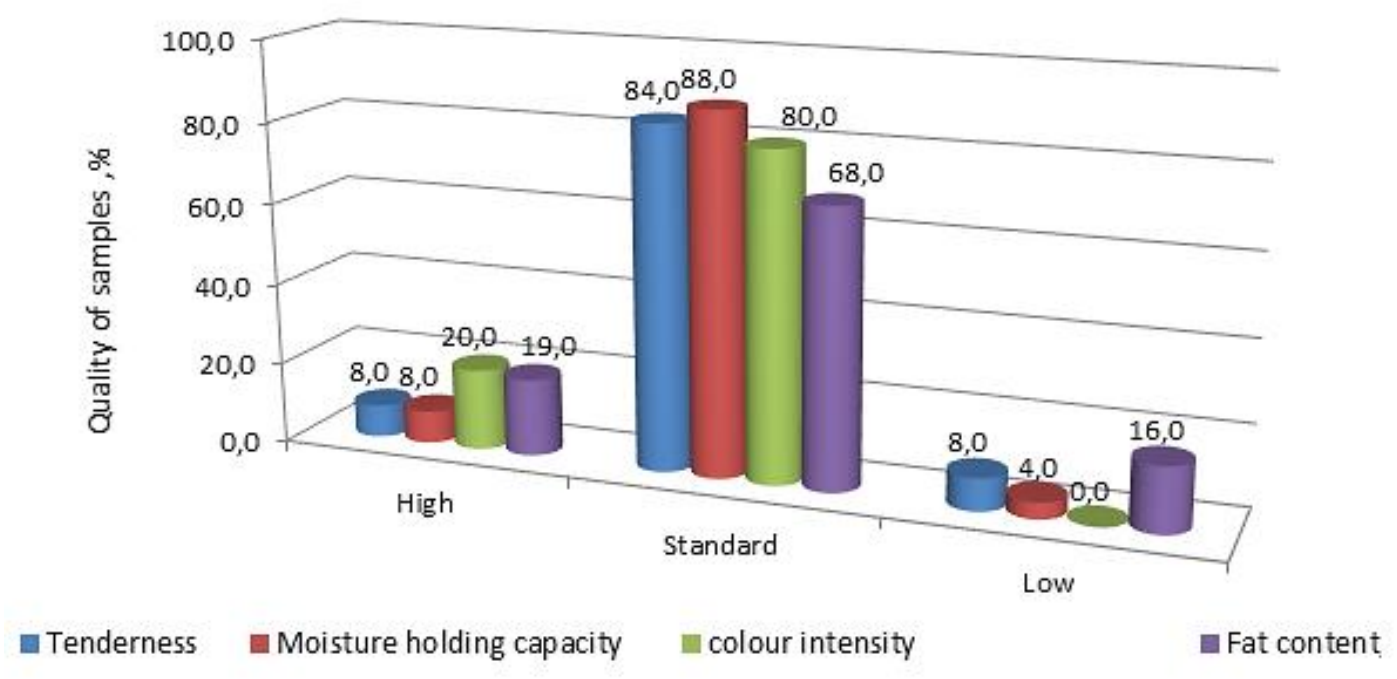

Fig. 1. The quality of muscle tissue of young pigs

We registered, that in the samples of Longissinus dorsi muscle the pH was 5.62, moisture holding capacity - $60.10 \%$; colour intensity -73.60 units of ext. $\times 1000$, tenderness -9.41 , crude fat $-2.28 \%$ (Table 1 ). The moisture content was $74.13 \%$, hygroscopic moisture - $1.47 \%$, air-dry matter - $27.25 \%$; crude ash - $1.13 \%$, crude protein $-22.36 \%$, crude fat - $2.28 \%$, calcium - $0.045 \%$, and phosphorus - $0.126 \%$. The loss of absolute mass of the muscle tissue sample during thermal processing was $22.36 \%$, and its energy value is $121.88 \mathrm{kcal}$. All the parameters had high variability, thus the $\mathrm{CV}$ of $\mathrm{pH}$ was 2.49 and $\mathrm{CV}$ of crude fat content was 
( $\mathrm{pH}$, acidity units) to $74.56 \%$. According to the scale of meat quality assessment (Polyvoda, 1976), the high quality samples in terms of moisture holding capacity was $12.0 \%$, colour intensity, units of ext. $\times 1000-16.0 \%$, tenderness $-12.0 \%$ and the crude fat content - $16.0 \%$ (Fig. 1).

Analysis of the data shows that the minimum values of $\mathrm{pH}-5.46$, colour intensity of 71.40 , tenderness of $8.97 \mathrm{~s}$, loss during thermal processing of $21.46 \%$, hygroscopic moisture of $1.38 \%$, air-dry matter of $26.84 \%$, crude ash of $1.07 \%$, crude protein of $20.71 \%$, calcium of $0.042 \%$, and phosphorus of $0.124 \%$ were characterized the samples of muscle tissue from the weaners with a total protein content of 58.67-66.30 g/l (distribution class M-).

Table 3. Longissinus dorsi muscle parameters

\begin{tabular}{|c|c|c|c|c|c|c|}
\hline \multirow{4}{*}{ Parameter } & \multicolumn{3}{|c|}{ total protein, g/l } & \multicolumn{3}{|c|}{ creatinine, $\mu \mathrm{mol} / /$} \\
\hline & $77.53-83.70$ & $67.40-74.45$ & $58.67-66.30$ & $171.06-194.23$ & $144.28-166.52$ & $102.59-137.86$ \\
\hline & $\mathrm{M}^{+}$ & $M^{0}$ & $\mathrm{M}^{-}$ & $\mathrm{M}^{+}$ & $M^{0}$ & $M^{-}$ \\
\hline & $4^{*}$ & 16 & 5 & 8 & 11 & 6 \\
\hline \multirow{2}{*}{$\mathrm{pH}^{\star *}$} & $5.63 \pm 0.06$ & $5.66 \pm 0.03$ & $5.46 \pm 0.08$ & $5.61 \pm 0.05$ & $5.60 \pm 0.05$ & $5.66 \pm 0.05$ \\
\hline & $2.03 \pm 0.72$ & $1.90 \pm 0.34$ & $3.08 \pm 0.97$ & $2.43 \pm 0.61$ & $2.98 \pm 0.64$ & $1.99 \pm 0.58$ \\
\hline moisture holding & $59.94 \pm 1.79$ & $59.46 \pm 1.36$ & $62.27 \pm 1.79$ & $59.02 \pm 1.22$ & $59.58 \pm 1.76$ & $62.84 \pm 1.91$ \\
\hline capacity, \% & $5.96 \pm 2.11$ & $9.16 \pm 1.62$ & $6.44 \pm 2.04$ & $5.83 \pm 1.46$ & $9.77 \pm 2.08$ & $7.48 \pm 2.16$ \\
\hline \multirow{2}{*}{ tenderness, s } & $9.92 \pm 1.02$ & $9.43 \pm 0.36$ & $8.97 \pm 0.41$ & $9.55 \pm 0.52$ & $8.95 \pm 0.34$ & $10.09 \pm 0.69$ \\
\hline & $20.46 \pm 7.23$ & $15.07 \pm 2.67$ & $10.30 \pm 3.269$ & $15.50 \pm 3.88$ & $12.77 \pm 2.72$ & $16.85 \pm 4.87$ \\
\hline \multirow{2}{*}{$\begin{array}{l}\text { colour intensity, units of } \\
\text { ext. } \times 1000\end{array}$} & $72.25 \pm 6.02$ & $74.62 \pm 2.57$ & $71.40 \pm 5.94$ & $70.50 \pm 3.85$ & $74.81 \pm 3.61$ & $75.50 \pm 3.60$ \\
\hline & $16.56 \pm 5.86$ & $13.75 \pm 2.43$ & $18.59 \pm 5.88$ & $15.46 \pm 3.87$ & $15.99 \pm 3.41$ & $11.69 \pm 3.38$ \\
\hline \multirow{2}{*}{$\begin{array}{l}\text { loss during thermal } \\
\text { processing, } \%\end{array}$} & $24.04 \pm 2.18$ & $21.71 \pm 0.81$ & $21.46 \pm 1.26$ & $21.58 \pm 1.31$ & $22.10 \pm 0.91$ & $22.49 \pm 1.61$ \\
\hline & $18.11 \pm 6.40$ & $15.00 \pm 2.65$ & $13.14 \pm 4.16$ & $17.13 \pm 4.28$ & $13.65 \pm 2.91$ & $17.47 \pm 5.05$ \\
\hline \multirow{2}{*}{ energy value, kcal } & $116.55 \pm 2.57$ & $121.50 \pm 2.56$ & $127.38 \pm 13.94$ & $119.80 \pm 1.65$ & $127.34 \pm 6.06$ & $114.68 \pm 5.59$ \\
\hline & $4.41 \pm 1.56$ & $8.41 \pm 1.49$ & $24.47 \pm 7.74$ & $3.90 \pm 0.98$ & $15.78 \pm 3.36$ & $11.93 \pm 3.45$ \\
\hline
\end{tabular}

${ }^{*}$ number of animals, ${ }^{* *}$ mean and SD, CV and $\mathrm{S}_{\mathrm{cv}}$.

Table 4. Chemical composition of Longissinus dorsi muscle

\begin{tabular}{|c|c|c|c|c|c|c|}
\hline \multirow{4}{*}{ Parameter } & \multicolumn{3}{|c|}{ total protein, g/l } & \multicolumn{3}{|c|}{ creatinine, $\mu \mathrm{mol} / \mathrm{l}$} \\
\hline & $77.53-83.70$ & $67.40-74.45$ & $58.67-66.30$ & $171.06-194.23$ & $144.28-166.52$ & $102.59-137.86$ \\
\hline & $\mathrm{M}^{+}$ & $M^{0}$ & $M^{-}$ & $\mathrm{M}^{+}$ & $M^{0}$ & $M^{-}$ \\
\hline & $4^{*}$ & 16 & 5 & 8 & 11 & 6 \\
\hline \multirow[t]{2}{*}{ total moisture ** } & $\begin{array}{c}73.93 \pm 0.38 * \\
\star\end{array}$ & $74.06 \pm 0.51$ & $74.54 \pm 1.65$ & $74.40 \pm 0.17$ & $73.44 \pm 0.81$ & $75.05 \pm 1.07$ \\
\hline & $1.03 \pm 0.37$ & $2.75 \pm 0.49$ & $4.95 \pm 1.57$ & $0.64 \pm 0.16$ & $3.67 \pm 0.78$ & $3.48 \pm 1.01$ \\
\hline \multirow{2}{*}{ hygroscopic moisture } & $1.64 \pm 0.08$ & $1.45 \pm 0.03$ & $1.38 \pm 0.14$ & $1.48 \pm 0.05$ & $1.46 \pm 0.06$ & $1.46 \pm 0.10$ \\
\hline & $9.67 \pm 3.43$ & $9.06 \pm 1.60$ & $22.35 \pm 7.07$ & $9.90 \pm 2.48$ & $13.84 \pm 2.95$ & $17.07 \pm 4.93$ \\
\hline \multirow{2}{*}{ air-dry matter } & $27.20 \pm 0.43$ & $27.39 \pm 0.49$ & $26.84 \pm 1.71$ & $27.07 \pm 0.14$ & $28.01 \pm 0.79$ & $26.07 \pm 1.11$ \\
\hline & $3.16 \pm 1.12$ & $7.27 \pm 1.29$ & $14.26 \pm 4.51$ & $1.47 \pm 0.37$ & $9.35 \pm 1.99$ & $10.38 \pm 3.0$ \\
\hline \multirow{2}{*}{ ash } & $1.16 \pm 0.02$ & $1.14 \pm 0.02$ & $1.07 \pm 0.05$ & $1.14 \pm 0.01$ & $1.12 \pm 0.04$ & $1.12 \pm 0.05$ \\
\hline & $3.92 \pm 1.39$ & $8.29 \pm 1.47$ & $11.40 \pm 3.61$ & $2.60 \pm 0.65$ & $10.33 \pm 2.20$ & $11.15 \pm 3.22$ \\
\hline \multirow{2}{*}{ protein } & $22.99 \pm 0.39$ & $22.72 \pm 0.49$ & $20.71 \pm 1.0$ & $22.40 \pm 0.26$ & $22.74 \pm 0.76$ & $21.61 \pm 0.91$ \\
\hline & $3.37 \pm 1.19$ & $8.57 \pm 1.52$ & $10.80 \pm 3.42$ & $3.23 \pm 0.81$ & $11.03 \pm 2.35$ & $10.25 \pm 2.96$ \\
\hline \multirow{2}{*}{ fat } & $1.40 \pm 0.17$ & $2.06 \pm 0.19$ & $3.67 \pm 1.54$ & $2.04 \pm 0.26$ & $2.68 \pm 0.72$ & $1.87 \pm 0.47$ \\
\hline & $23.97 \pm 8.50$ & $36.97 \pm 6.54$ & $93.49 \pm 2.96$ & $35.37 \pm 8.84$ & $88.43 \pm 18.86$ & $61.20 \pm 17.69$ \\
\hline \multirow{2}{*}{ calcium (Ca) } & $0.05 \pm 0.0$ & $0.045 \pm 0.0$ & $0.042 \pm 0.0$ & $0.046 \pm 0.0$ & $0.045 \pm 0.0$ & $0.042 \pm 0.0$ \\
\hline & $12.50 \pm 4.43$ & $11.84 \pm 2.10$ & $13.88 \pm 4.39$ & $9.95 \pm 2.49$ & $13.19 \pm 2.81$ & $13.12 \pm 3.79$ \\
\hline \multirow{2}{*}{ phosphorus (P) } & $0.13 \pm 0.01$ & $0.124 \pm 0.01$ & $0.124 \pm 0.0$ & $0.115 \pm 0.01$ & $0.134 \pm 0.01$ & $0.124 \pm 0.01$ \\
\hline & $21.64 \pm 7.67$ & $16.16 \pm 2.86$ & $26.95 \pm 8.53$ & $14.01 \pm 3.50$ & $17.12 \pm 3.65$ & $23.82 \pm 6.88$ \\
\hline
\end{tabular}

${ }^{*}$ number of animals, ${ }^{* *}$ mean and SD, CV and $\mathrm{S}_{\mathrm{cv}}$.

We did not reveal certain pattern in the qualitative composition of muscle towards concentration of creatinine in the serum. Thus, the maximum indicators of moisture holding capacity is $62.84 \%$, colour intensity -75.50 units of ext. $\times 1000$, tenderness $10.09 \%$, loss during thermal processing - $22.49 \%$, total moisture content $-75.05 \%$ and hygroscopic moisture $-1.46 \%$; they were found in class $\mathrm{M}^{-}$animals (serum creatinine concentrations fluctuate from 102.59 to $137.86 \mu \mathrm{mol} / \mathrm{l}$ ).

Indicators of muscle tissue samples of class $\mathrm{m}^{0}$ animals with serum creatinine concentration ranged from 144.28 to 166.52 $\mu \mathrm{mol} / \mathrm{l}$; they were characterized by a higher energy value - $127.34 \mathrm{kcal}$, air-dry matter content - 28.01\%, protein - $22.74 \%$, fat $2.68 \%$ and phosphorus $-0.134 \%$. Young pigs of class $\mathrm{M}^{+}$(serum creatinine concentration ranged from 171.06 to $194.23 \mathrm{mmol} / \mathrm{l}$ ) outclassed the weaners from $\mathrm{M}^{0}$ and $\mathrm{M}^{-}$in ash content by $0.02(\mathrm{td}=0.55, \mathrm{P}>0.05)$ and $0.02 \%(\mathrm{td}=0.39, \mathrm{P}>0.05)$, respectively; 
in calcium content by $0.001(\mathrm{td}=0.42, \mathrm{P}>0.05)$ and $0.004 \%(\mathrm{td}=1.44, \mathrm{P}>0.05)$, respectively.

Table 5. Correlation between physicochemical properties and chemical composition of Longissinus dorsi muscle (Large White weaners, $n=25$ )

\begin{tabular}{|c|c|c|c|c|c|}
\hline Parameter & $\mathrm{pH}$ & $\begin{array}{c}\text { moisture holding } \\
\text { capacity }\end{array}$ & tenderness & colour intensity & $\begin{array}{c}\text { loss during } \\
\text { thermal } \\
\text { processing }\end{array}$ \\
\hline \multirow{2}{*}{ total moisture } & $0.12 \pm 0.21$ & $0.29 \pm 0.19$ & $0.18 \pm 0.21$ & $0.32 \pm 0.19$ & $0.13 \pm 0.21$ \\
\hline & $0.58 *$ & 1.49 & 0.87 & 1.61 & 0.62 \\
\hline \multirow{2}{*}{$\begin{array}{l}\text { hygroscopic } \\
\text { moisture }\end{array}$} & $0.18 \pm 0.21$ & $0.40 \pm 0.19 *$ & $0.13 \pm 0.21$ & $0.11 \pm 0.21$ & $-0.05 \pm 0.21$ \\
\hline & 0.88 & 2.11 & 0.60 & 0.53 & 0.24 \\
\hline \multirow{2}{*}{ air-dry matter } & $-0.12 \pm 0.21$ & $-0.28 \pm 0.20$ & $0.15 \pm 0.21$ & $-0.30 \pm 0.19$ & $-0.12 \pm 0.21$ \\
\hline & 0.58 & 1.39 & 0.72 & 1.51 & 0.57 \\
\hline \multirow{2}{*}{ ash } & $-0.12 \pm 0.21$ & $-0.36 \pm 0.19$ & $0.06 \pm 0.21$ & $0.23 \pm 0.20$ & $0.08 \pm 0.21$ \\
\hline & 0.60 & 1.86 & 0.28 & 1.11 & 0.38 \\
\hline \multirow{2}{*}{ protein } & $-0.05 \pm 0.21$ & $-0.48 \pm 0.18^{*}$ & $-0.09 \pm 0.21$ & $-0.21 \pm 0.20$ & $0.08 \pm 0.21$ \\
\hline & 0.26 & 2.65 & 0.41 & 1.02 & 0.38 \\
\hline \multirow{2}{*}{ fat } & $-0.11 \pm 0.21$ & $0.18 \pm 0.21$ & $-0.14 \pm 0.21$ & $-0.15 \pm 0.21$ & $-0.25 \pm 0.21$ \\
\hline & 0.52 & 0.85 & 0.76 & 0.73 & 1.24 \\
\hline \multirow{2}{*}{ calcium } & $-0.16 \pm 0.21$ & $-0.31 \pm 0.19$ & $0.02 \pm 0.21$ & $-0.02 \pm 0.21$ & $0.06 \pm 0.21$ \\
\hline & 0.78 & 1.56 & 0.10 & 0.10 & 0.30 \\
\hline \multirow{2}{*}{ phosphorus } & $-0.21 \pm 0.20$ & $0.06 \pm 0.21$ & $-0.25 \pm 0.21$ & $0.13 \pm 0.21$ & $0.16 \pm 0.21$ \\
\hline & 1.01 & 0.29 & 1.26 & 0.63 & 0.75 \\
\hline
\end{tabular}

$\mathrm{P}<0.05, * \mathrm{r} \pm \mathrm{Sr}, \operatorname{tr}(\mathrm{r} \pm \mathrm{Sr}$ - correlation coefficient and semi-partial correlation)

Table 6. Correlations between chemical composition of Longissinus dorsi muscle and blood serum parameters (Large White weaners, $n=25$ )

\begin{tabular}{lcccc}
\hline Parameter & \multicolumn{2}{c}{ total protein } & \multicolumn{2}{c}{ creatinine } \\
& $r \pm S r$ & $\operatorname{tr}$ & $\mathrm{r} \pm \mathrm{Sr}$ & 0.72 \\
$\mathrm{PH}$ & $0.29 \pm 0.19$ & 1.44 & $-0.15 \pm 0.21$ & $-0.18 \pm 0.21$ \\
moisture holding capacity & $-0.12 \pm 0.21$ & 0.59 & $-0.04 \pm 0.21$ & 0.87 \\
tenderness & $0.22 \pm 0.20$ & 1.06 & $-0.05 \pm 0.21$ & 0.19 \\
colour intensity & $0.03 \pm 0.21$ & 0.16 & $-0.02 \pm 0.21$ & 0.24 \\
loss during thermal rocessing & $0.08 \pm 0.21$ & 0.38 & $0.06 \pm 0.21$ & 0.07 \\
total moisture & $-0.19 \pm 0.20$ & 0.91 & $-0.08 \pm 0.21$ & 0.31 \\
hygroscopic moisture & $0.36 \pm 0.19$ & 1.87 & $-0.03 \pm 0.21$ & 0.37 \\
air-dry matter & $0.17 \pm 0.21$ & 0.84 & $0.03 \pm 0.21$ & 0.13 \\
Ash & $0.27 \pm 0.20$ & 1.33 & $0.14 \pm 0.21$ & 0.16 \\
Protein & $0.37 \pm 0.19$ & 1.89 & $-0.18 \pm 0.21$ & 0.70 \\
Fat & $-0.26 \pm 0.21$ & 1.28 & $0.24 \pm 0.20$ & 0.87 \\
Calcium & $0.38 \pm 0.19$ & 1.94 & $-0.22 \pm 0.20$ & 1.17 \\
Phosphorus & $0.14 \pm 0.21$ & 0.65 & & 1.10 \\
\hline
\end{tabular}

$\mathrm{r} \pm \mathrm{Sr}$ - correlation coefficient and semi-partial correlation

\section{Conclusions}

We revealed that the biochemical parameters of weaners serum - total protein and creatinine concentration were within the physiological norm. The number of samples of Longissinus dorsi muscle of high quality in terms of moisture holding capacity is $12 \%$, fat content $-16 \%$, tenderness $-12 \%$ and colour intensity $-16 \%$. We did not register a significant correlation between the physicochemical properties and chemical composition of We determined significant correlations between moisture holding capacity and protein content $(r=-0.48, \mathrm{tr}=2.65)$, moisture holding capacity and hygroscopic moisture content $(r=0.40, \mathrm{tr}=$ 2.11). The correlation between total protein in blood serum and fat content in muscle tissue was -0.27 (tr $=1.28$ ), whereas the correlation between total protein content in serum and calcium content in muscle tissue was 0.38 (tr $=1.94$ ), but this was insignificant. 
Aknevskyy, Yu.P., Buslyk, T.V, Hryshyna, L.P., Balatskyy, V.M. (2013). Vplyv polimorfizmu henu retseptora melanokortynu - 4 (MS4R) na vidhodivelni ta myasni yakosti pomisnykh, hibrydnykh i chystoporidnykh svyney velykoyi biloyi porody. Svinarstvo, 63, 28-37 (In Ukrainian).

Allison, R.D., Laven, R.A. (2000). Effect of vitamin E supplementation on the health and fertility of dairy cows: a review. Vet. Rec, 147(25), 703-708.

Balatskii,V.M., Korinnii, S.M., Bankovska, I.B., Hibolenko, O.S. (2015). Asotsiatsiia henu relizing-faktora hormone rostu za yakistiu miasa svynei velykoi biloi porody ukrainskoi selektsii. Svynarstvo: mizhvid. temat. nauk. zb. Poltava, 67, 107-112 (In Ukrainian).

Balatsky, V., Oliinychenko, Y., Sarantseva, N., Getya, A., Saienko, A., Vovk, V., Doran O. (2018). Association of single nucleotide polymorphisms in leptin $(L E P)$ and leptin receptor $(L E P R)$ genes with backfat thickness and daily weight gain in Ukrainian Large White pigs. Livestock Science, 217, 157-161 (In Ukrainian).

Bankovskaya, I. B., Voloshchuk, V. M. (2015). Morfologicheskiy sostav chastey tush sviney v zavisimosti ot genotipa i sposoba soderzhaniya. Zootekhnicheskaya nauka Belarusi: sb. nauch. tr. Zhodino, 50(2), 140-146. (In Russian).

Bazhov, G.M., Komlatskiy, V.I. (1989). Biotekhnologiya intensivnogo svinovodstva. Moscow. Rosagropromizdat (In Russian).

Berezovskyy, M.D., Khatko, I.V. (2005) Metodyky otsinky knuriv i svynomatok za yakistyu potomstva v umovakh pleminnykh zavodiv i pleminnykh reproduktoriv. Suchasni metodyky doslidzhen u svynarstvi. Poltava. (In Ukrainian).

Christianson D.W. (1991) Structural biology of zinc. Adv Protein Chem, 42, 281-355. doi: 10.1016/s0065-3233(08)60538-0.

Eidrigevich, E.V., Rayevskaya, V.V. (1966). Interyer selskokhozyaystvennykh zhivotnykh. Moscow. Kolos (In Russian).

Fedorenkova, L.A., Batkovskaya, T.V., Yanovich, Ye.A. (2012) Fiziko-khimicheskiye svoystva i organolepticheskaya otsenka myasa i sala razlichnykh genotipov sviney. Vestsi Natsyyanalnay akademii navuk Belarusi. (Seryya agrarnykh navuk), 3, 63-68. (In Russian).

Hryban, V.H., Chumak, V.O., Nemyrovskyy, V.I. (2001). Klinichna biokhimiya tvaryn. Dnipropetrovsk (In Ukrainian).

Kabanov, V. (2009). Biologicheskiye osnovy povysheniya intensivnosti svinovodstva Svinarstvo, 2, $27-28$ (In Russian).

Kamyshnikov, V.S. (2002) Spravochnik po kliniko-biokhimicheskoy laborotornoy diagnostike. Minsk. Belarus (In Russian).

Khalak, V., Gutyj, B., Bordun, O., Ilchenko, M., Horchanok, A. (2020a). Effect of blood serum enzymes on meat qualities of piglet productivity. Ukrainian Journal of Ecology, 10 (1), 158-161. doi: 10.15421/2020_25.

Khalak, V., Gutyj, B., Bordun, O., Horchanok, A., Ilchenko M., ... Lytvyshchenko, L. (2020b). Development and reproductive qualities of sows of different breeds: innovative and traditional methods of assessment. Ukrainian Journal of Ecology, 10(2), 356-360. doi: 10.15421/2020_109.

Kozyr, V., Khalak, V., Povod, M. (2019). DNA-type results swine for MS4R-gene and its association with productivity. Agrolife: Scientific journal. University of Agronomic Sciences and Veterinari Medicine of Bucharest. Bucharest, 8(1), 128-133.

Kramarenko, A.S., Karatieieva, O.I., Lykhach, A.V., Lugovoy, S.I., Lykhach, V.Ya., ... Kramarenko, S.S. (2019). Assessing genomic taurine/ zebuine admixture in the Southern Meat cattle based on microsatellite markers. Ukrainian Journal of Ecology, 9(1), $251-261$.

Kramarenko, S.S., Lugovoy, S.I., Kharzinova, V.R., Lykhach, V., Kramarenko, A.S., Lykhach, A.V. (2018). Genetic diversity of Ukrainian local pig breeds based on microsatellite markers. Regulatory Mechanisms in Biosystems, 9(2), 177-182. doi:10.15421/021826.

Lakin, G. F. (1990). Biometriya. Moscow. Vysshaya shkola. (In Russian).

Levchenko, V.I., Vlizlo, V.V., Kondrakhin, I.P., Melnychuk, D.O., Apukhovska, L.I.,... Tsvilikhovskii, V.I. (2002). Veterynarna klinichna biokhimiia. Bila Tserkva (In Ukrainian).

Magomedaliev, I.M., Nekrasov, R.V., Chabaev, M.G., Dzhavakhiya, V.V., Glagoleva, E.V., Kartashov, M.I., Durnikin, D.A., \& Matsyura, A.V. (2019). Use of different concentrations of enzymesporin probiotic in feeding of growing young pigs. Ukrainian Journal of Ecology, 9, 704-708. Doi: 10.15421/2019_813.

Metodicheskiye rekomendatsii po otsenke myasnoy produktivnosti, kachestva myasa i podkozhnogo zhira sviney. (1987). Moscow. (In Russian).

Polivoda, A.M., Strobykina, R.V., Lyubetskiy, M.D. (1977). Metodika otsenki kachestva produktsii uboya u sviney. Metodiki issledovaniy po svinovodstvu. Kharkov (In Russian).

Polyvoda, A.M. (1976) Otsinka yakosti svynyny za fizyko-khimichnymy pokaznykamy. Svynarstvo, 24, 57-62 (In Ukrainian).

Rybalko, V.P., Bankovskaya, I.B., Getya, A.A. (2010). Znacheniye otsenki vkusovykh kachestv myasa i sala sviney v selektsionnoy praktike. Proceed. XVII Int. Sc. Conf. Sovremennyye problemy intensifikatsii proizvodstva svininy v stranakh SNG. 7-10.07.2010. Ulyanovsk, 276-280 (in Russian).

Susol, R.L. (2015). Metodolohiya stvorennya i vykorystannya novykh henotypiv svyney vitchyznyachnoho ta zarubizhnoho pokhodzhennya v umovakh pivdnya Ukrayiny. Thesis of Doctoral Dissertation. Mykolayiv (In Ukrainian).

Tatulov, Yu.V. (2009). Faktory, opredelyayushchiye myasnuyu produktivnost i kachestvo svininy. Myasnyye tekhnologii, 12, 38-39. (In Russian).

Talilov, Yu.V. (2009). Faktory, opredeliaiushchiie miasnuiu produktivnost I kachestvo. Miasniie tekhnolohii, 12, 38-39. (In Russian).

Tserenyuk, O.M. (2018). Metodolohiya vyznachennya efektu heterozysu v svynarstvi. Scientific and technical bulletin of IL NAAS, 119, 173-184. (In Ukrainian).

Tserenyuk, O.M. (2017). Vidhodivelni oznaky molodnyaku svyney z riznoyu stresostiykistyu v period "kryzy vidluchennya". Scientific and technical bulletin of IL NAAS, 118, 191-199. (In Ukrainian).

Vlizlo, V.V., Fedoruk, R.S., Ratych, I. B., Vishchur, O.I., Sharan, M.M., ... Martyn, Yu.V. (2012). Laboratorni metody doslidzhen u biolohii, tvarynnytstvi ta veterynarnii medytsyni. Lviv. SPOLOM. (In Ukrainian).

\section{Citation}

Khalak, V., Horchanok, A., Kuzmenko, O., Lytvyshchenko, L., Lieshchova, M., Kalinichenko, A., Liskovich, V., Zagoruy, L. (2020). Protein metabolism, physicochemical properties and chemical composition of muscle tissue in Large White weaners.

Ukrainian Journal of Ecology, 10(4), 127-131.

(cc) EY This work is licensed under a Creative Commons Attribution 4.0. License 